\title{
Sequence effects of combined resistance exercises with step choreography in the same session in women's oxygen uptake during and postexercise
}

\author{
José Vilaça-Alves ${ }^{1,2}$, Ana Regado ${ }^{1}$, Daniel Marinho ${ }^{2,3}$, Eduardo Borba Neves ${ }^{4}$, Claudio Rosa ${ }^{1}$, \\ Francisco Saavedra, ${ }^{1,2}$ and Victor M. Reis ${ }^{1,2}$ \\ ${ }^{1}$ Sport Sciences Department, University of Trás-os-Montes e Alto Douro, Vila Real, Portugal, ${ }^{2}$ Research Centre in Sports Sciences, Health Sciences and Human \\ Development, CIDESD, Vila Real, Portugal, ${ }^{3}$ Sports, University of Beira Interior, Guarda, Portugal and ${ }^{4}$ Graduate Program of Biomedical Engineering, \\ Universidade Tecnologica Federal do Parana, Curitiba, Brazil
}

\section{Summary}

\section{Correspondence \\ José Vilaça-Alves, Sport Sciences Department, University of Trás-os-Montes e Alto Douro, Quinta de Prados, Complexo Desportivo, 5000-801 Vila real, Portugal \\ E-mail: josevilaca@utad.pt}

\section{Accepted for publication \\ Received 20 March 2016; \\ accepted 6 June 2016}

\section{Key words}

concurrent training; energy expenditure; exercise order; oxygen consumption; women
The combination of step choreography (SC) with resistance training exercises $(\mathrm{RE})$ in the same session is common in class fitness rooms populated mainly by women to increase energy expenditure. The aim of this study was to evaluate the differences in the exercise oxygen uptake and postexercise between two different combinations of resistance training exercises and step choreography, regarding the order of execution. Thirteen active women (30.31 \pm 4.42 years, $62.02 \pm 5.37 \mathrm{~kg}, 162.65 \pm 4.40 \mathrm{~cm}, 19 \cdot 14 \pm 3.29 \%$ body fat) performed two combinations: step choreography before resistance training, where resistance training was divided into two blocks of analysis (10 min each); and step choreography divided into three equal blocks (10 min for each block), before, in the middle and after resistance exercise. There were significant differences $(P<0.05)$ between the two sessions in oxygen uptake postexercise in the period of 0-5 min. A significant increase $(P<0 \cdot 0001)$ in the oxygen uptake absolute and relative in the heart rate between blocks 1 and 2 of resistance exercise in the two sessions was observed. In the step choreography in blocks, a significant $(P=0.001)$ decrease between blocks 2 and 3 in the step choreography before resistance exercise and a significant $(P<0.05)$ increase in the heart rate in both sessions between blocks were observed. The combination of step choreography and resistance exercises during the same exercise session is a good strategy to promote an elevation of women's oxygen uptake during and after an exercise session, independent of the sequence used.

\section{Introduction}

The combination of endurance exercise (EE) and resistance exercise (RE) in the same session is commonly called concurrent training $(\mathrm{CT})$. It is very common in physical fitness training programmes (ACSM, 2013); especially when the aim is to increase energy expenditure during and after an exercise session (Alves et al., 2012), this combination is often due to lack of available time for exercise (Rosa et al., 2015). The literature presents positive chronic effects on body composition with the combination of EE with RE during the same session (Dolezal \& Potteiger, 1998; Davitt et al., 2014). Positive improvements in the basal metabolic rate (Dolezal \& Potteiger, 1998; Davitt et al., 2014) and fat loss (LeMura et al., 2000; Leveritt et al., 2003; Ghahramanloo et al., 2009) are also described as resulting from $\mathrm{CT}$.
The manipulation of the sequence of the EE and RE affects the acute and chronic responses to CT (McCarthy et al., 1995; Chtara et al., 2005; Cadore et al., 2012; Schumann et al., 2013). However, the sequence of combining the two types of exercise may also be an important way to maximize benefits of the concurrent training to promote energy expenditure. However, the influence of the $\mathrm{EE}$ and $\mathrm{RE}$ sequence on volume of oxygen consumed per min $\left(\mathrm{VO}_{2}\right)$ is scarcely reported in the literature (Oliveira \& Oliveira, 2011; Alves et al., 2012; Davitt et al., 2014).

Alves et al. (2012) showed no significant differences between different combinations of $\mathrm{EE}$ and RE exercises, but found a higher $\mathrm{VO}_{2}$ in the 5- to 15-min postexercise period when EE was performed before RE and in the 10-15 min when the EE was performed in the middle of RE blocks.

Drummond et al. (2005) observed a higher $\mathrm{VO}_{2}$ on the $\mathrm{EE}$ when it was performed after RE, and Di Blasio et al. (2012) 
observed that for women, higher postexercise $\mathrm{VO}_{2}$ values were present when the EE was divided into three blocks of 10 min between blocks of RE. Thus, if the EE is divided into blocks during $\mathrm{RE}$, it is likely the $\mathrm{VO}_{2}$, during exercise, will be higher than when the EE is performed continuously.

Most women look for physical activities that lead to high energy expenditure aimed at burning fat. Classes of step choreography (SC) are popular because when it is combined with the RE exercises such classes can provide higher energy expenditure. Hence, it seems important to observe the effects of different combinations of $\mathrm{SC}$ with $\mathrm{RE}$ on the $\mathrm{VO}_{2}$ during and postexercise. The aim of this study was to evaluate the differences in the oxygen uptake between two different combinations of RE and SC, regarding the order of execution.

\section{Methods}

Thirteen active women (30.31 \pm 4.42 years, $62.02 \pm$ $5.37 \mathrm{~kg}, \quad 162.65 \pm 4.40 \mathrm{~cm}, \quad 19 \cdot 14 \pm 3.29 \%$ body fat) started and completed all the established procedures for this study. All participants completed a physical activity and medical history questionnaire. The volunteers were engaged in physical activity (step and body pump group classes and weight training) for at least 3 day week ${ }^{-1}$ over the past year. Additionally, the participants were informed of the possible risks or discomfort involved in the experiment and provided written informed consent to participate in the study. The procedures were designed according to the Helsinki Declaration and were approved by Research Ethics Committee of the Institution $n^{\circ} 15 /$ CIDESD-UTAD/2014.

The session total load and exercise intensity of both RE and SC were designed to stimulate a high $\mathrm{VO}_{2}$, to involve larger muscle groups and to meet the time available for training in modern societies. Participants performed the third and fourth sessions in a randomized order with a 7-day recovery period between sessions. Sessions were conducted at the same time of the day. In addition, all women were instructed to refrain from any strenuous physical activity for $48 \mathrm{~h}$ (step, body pump, weight training and recreational activities) prior to testing and to record and duplicate their meals for $24 \mathrm{~h}$ before the third and fourth sessions.

The 1RM testing protocol was described previously by Kraemer et al. (1995). The subjects, before of the strength testing, were familiarized with the RE exercises over four visits, during 2 weeks and with a weekly frequency of two times. After the familiarization, the $1 \mathrm{RM}$ tests were performed for half-squat, bench press, lunge and lat row with a bar, in this exercise order, with an interval of $10 \mathrm{~min}$ between tests. The heaviest load achieved in the test and retest sessions was considered the $1 \mathrm{RM}$ load. To minimize the error during the $1 \mathrm{RM}$ tests and retest, the following strategies were adopted: (i) standardized instructions concerning the testing procedures were given to participants before the test sessions; (ii) participants received standardized instructions on exercise technique; (iii) standard verbal encouragement was provided during the testing procedure; and (iv) the velocity of the movements were controlled by an electronic metronome (Korg MA-30, Korg, Melville, New York, USA). The $1 \mathrm{RM}$ was determined in five attempts with a rest interval of 5 min between each attempt.

The $\mathrm{VO}_{2}$ before, during and after the two different sequences was measured continuously by a portable gas analyser (Cosmed $^{\circledR} \mathrm{K} 4 \mathrm{~b}^{2}$, Rome, Italy), in which the gas samples were collected and measured every $10 \mathrm{~s}$. The respiratory exchange ratio (RER), volume of oxygen consumed per min $\left(\mathrm{VO}_{2}\right)$ and volume of carbon dioxide produced per min $\left(\mathrm{VCO}_{2}\right)$ were standardized and calculated prior to each examination. The HR was continuously monitored (Polar Wireless Electrode double, Kempele, Finland). Ambient temperature and humidity were between $20^{\circ} \mathrm{C}$ and $25^{\circ} \mathrm{C}$ and $40 \%$ and $65 \%$, respectively, for all the tests. The gas analyser was calibrated before each examination according to the manufacturer's guidelines. In the $24 \mathrm{~h}$ before an exercise session day, the subjects were required to (i) avoid caffeine or other metabolic altering supplements and drugs; (ii) engage in no physical activity; (iii) stay well hydrated and not change their habitual diet; and (iv) be well rested.

The participants recorded their food consumption during the day before the first exercise session and were asked to replicate it the day before the other session. The last meal of the day before testing was eaten not later than 20:00 h.

On testing days, $30 \mathrm{~min}$ before exercise and after the measurement of the resting metabolic rate (RMR), all the subjects received a standardized meal aimed to balance the caloric intake among all the subjects. The meal consisted of $330 \mathrm{ml}$ of water, $350 \mathrm{ml}$ of orange juice and 35-g energy bar Pro-Plex (All-Stars Fitness products, Peibenberg, Germany), amounting to $150 \mathrm{Kcal}(6.3 \mathrm{~g}$ protein, $12.6 \mathrm{~g}$ carbohydrate and $8.1 \mathrm{~g}$ fat).

Before each exercise session and after a minimum of $12 \mathrm{~h}$ of fasting, the RMR was measured by indirect calorimetry using Cosmed ${ }^{\circledR} \mathrm{K} \mathrm{b}^{2}$. The measurement was performed in an isolated room, with the door closed and the lights dimmed. The RMR was measured for $30 \mathrm{~min}$. The RMR was determined from steady-state $\mathrm{VO}_{2}$ values during the last $25 \mathrm{~min}$ of measurement. Immediately postexercise, the subjects returned to the room where the RMR measurement was repeated.

During each session, expired gases were continuously measured breath by breath with Cosmed ${ }^{\circledR} \mathrm{K} 4 \mathrm{~b}^{2}$ and then averaged as 20 -s intervals. HR was also measured continuously using an HR monitor (Polar Wireless Double Electrode T31, Kempele, Finland).

All participants performed two experimental sessions: (i) 30 min of SC followed by four RE exercises (SCRE) and (ii) three blocks of SC (10 min each) with two blocks of RE among them (SRSRS). In RE block 1, three sets of 10 repetitions in half-squat and bench exercises, with a 60-s rest interval between sets and load of $70 \%$ of $1 \mathrm{RM}$, were performed. In RE block 2, lunge and lat row barbell exercises with same methodology as block 1 were performed. After observing whether participants met the inclusion criteria, they were familiarized with the $\mathrm{SC}$ and the $\mathrm{RE}$ over the 


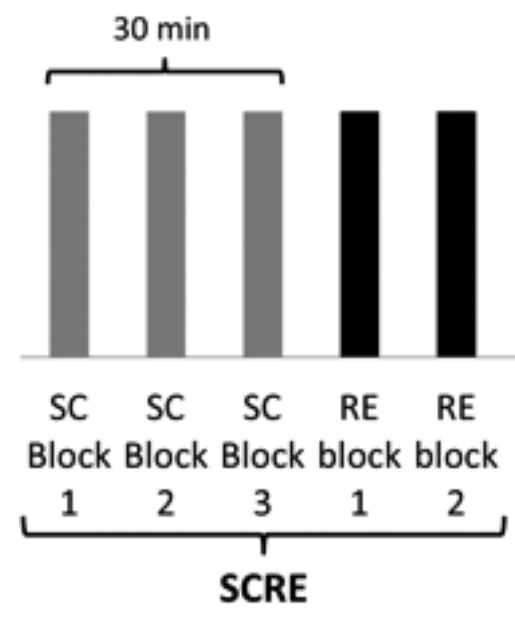

Figure 1 Diagram of exercises sessions

course of 2 weeks. After that, the participants were evaluated in four sessions. Session 1 was dedicated to the measurement of height, body mass, estimated body fat and 1repetition maximum test $(1 \mathrm{RM})$. In session 2, with an interval of $72 \mathrm{~h}$ from the last session, the retest of the $1 \mathrm{RM}$ was performed. Sessions 3 and 4 were performed with an interval of 7 days between them, where the $\mathrm{VO}_{2}$, heart rate (HR) and respiratory exchange ratio (RER) were measured $30 \mathrm{~min}$ before, during the exercise and $30 \mathrm{~min}$ after the two exercise sessions and followed the format (Fig. 1): $30 \mathrm{~min}$ of SC before RE (SCRE) and SC divided into three equal blocks [before, in the middle and after $\mathrm{RE}$ (SRSRS)].

Resistance training exercises: the participants performed, in this sequence, in the two sessions, the half-squat, bench press, lunge and lat row barbell exercises with a load of $70 \%$ of 1RM. These exercises were chosen to elicit a large muscle mass in upper and lower limbs. Each exercise session consisted of three sets of 10 repetitions performed with a 60-s rest interval between sets and at a cadence of 60 beats per min (b.m ${ }^{-1}$ ), which was controlled by an electronic metronome (Korg MA-30, Korg, Melville, New York, USA).

Endurance exercise: the SC was performed using a step platform for $30 \mathrm{~min}$ or divided into three blocks of $10 \mathrm{~min}$ each. Step aerobics combinations include patterns such as conventional basic step; 'V' step; 'L' step to both left and right side; turn step (right and left lead); and alternating leg up, up, down, down patterns. The arm movements were incorporated simultaneously with selected steps. The music was always the same in all sessions and with a cadence of 120 foot strikes per min.

During each session, the subject's HR was monitored using a HR monitor (Polar Wireless Double Electrode T31, Kempele, Finland) to ensure the subject performed the aerobic exercise at their individual target HR zone. The HR zone was determined using the heart rate reserve (HRR) and was establish between $60 \%$ and $80 \%$ of HRR. HRR was determined by Karvonen \& Vuorimaa (1988)

\section{Statistical analysis}

Normality and sphericity assumptions were confirmed with the Shapiro-Wilk test and Mauchly's test, respectively. Results are presented as mean \pm SD. Differences between session's values during the exercise were investigated with a univariate ANOVA. The differences within and between session's of the values of resting and postexercise $\mathrm{VO}_{2}$ were investigated with the ANOVA for repeated measures, with post hoc Bonferroni. The effect size was calculated by the partial eta square $\left(\eta_{\mathrm{p}}{ }^{2}\right)$ (Cohen, 2013). The significance level was maintained at $P<0 \cdot 05$.

\section{Results}

The values regarding the performance of the participants during the $1 \mathrm{RM}$ and reliability for each exercise with intraclass correlation coefficients (ICC) for half-squat $(51.15 \pm 14.74$; ICC $>0.99)$, bench press $(37.69 \pm 13.48$; ICC $>0.98)$, lunge $(58.85 \pm 21.42 ;$ ICC $>0.99)$ and lat row barbell $(42.31$ $\pm 10 \cdot 33$; ICC $>0.99)$ were observed. No significant differences were observed on the absolute $(1.44 \pm 0.22$ versus $1.56 \pm 0 \cdot 26)$ and relative $\mathrm{VO}_{2}(23.85 \pm 3 \cdot 17$ versus $25 \cdot 41 \pm$ $3.60)$, on HR $(156.68 \pm 12.93$ versus $155.55 \pm 11.27)$ or on RER $(1.00 \pm 0.03$ versus $1.03 \pm 0.03)$ between the two exercise orders, SCRE and SRSRS, respectively.

A significant time effect was observed in the $\mathrm{VO}_{2}$ and $\mathrm{HR}$ values during the $\mathrm{SC}$ moments. There was an observed significant decrease in the absolute $(P=0.001)$ and relative $\mathrm{VO}_{2}$ $(P=0.002)$ between the SC block 2 and SC block 3 in the SCRE session and a significant increase in the HR values between the SC blocks 1 and $3 \quad(P<0.0001)$ in SCRE $(P<0.0001)$ and among all blocks in the SRSRS session $(P<0.001)$. No significant differences were found in SC between sessions for any variable (Table 1).

In the RE, all variables increased significantly between blocks 1 and 2 in the two session's format, with exception of the RER value which did not change significantly. No significant 
differences were observed between sessions in any variable (Table 2).

There were significant differences regarding the time effect $\left(\mathrm{F}_{(6.144)}=432.875 ; P<0.0001 ; \mu_{\mathrm{p}}{ }^{2}=0.947\right)$ in the two sessions, namely the resting $\mathrm{VO}_{2}$ is significantly lower when compared to all the recovery moments $(P<0.05)$, and significant differences in the time effect $\mathrm{x}$ session $\left(\mathrm{F}_{(6.144)}=6.023\right.$; $\left.P=0.015 ; \quad \mu_{\mathrm{p}}{ }^{2}=0.201\right)$ were also observed. The single moment with significant differences between sessions was found in postexercise $\mathrm{VO}_{2}$ at 0-5 min (Table 3).

\section{Discussion}

The main finding observed in the present study was that the division of SC into three blocks did not significantly influence the $\mathrm{VO}_{2}$, HR and a RER compared to performing SC continuously. During recovery $0-5 \mathrm{~min}$, there was an observed significant difference between sessions in the $\mathrm{VO}_{2}$. This small difference was, however, not likely to promote meaningful physiological implications (e.g. weight loss). Thus, it is possible to divide the endurance exercise between resistance exercises without causing limitations to the cardiovascular demands.

The observed results corroborate with that in our previous study (Alves et al., 2012) which reported no influence of the $\mathrm{EE}$ and $\mathrm{RE}$ sequence in the mean $\mathrm{VO}_{2}$ during a training session. In addition, the relative $\mathrm{VO}_{2}$ in both studies was identical in the EE and RE, although the absolute $\mathrm{VO}_{2}$ was higher (Alves et al., 2012). As the present study analysed women and the sample studied by Alves et al. (2012) was men, sex may explain the mismatch between the two studies. In fact, men's body mass is usually higher than women's and absolute $\mathrm{VO}_{2}$ is strongly related to body mass.

When we observed the $\mathrm{VO}_{2}$ during the $\mathrm{SC}$, our data did not corroborate findings reported by Drummond et al. (2005) and by Kang et al. (2009) which observed higher values of $\mathrm{VO}_{2}$ during treadmill run and cycle, respectively, when the RE was performed before aerobics. Differently, the results showed in Alves et al. (2012) and those presented in this study show that the $\mathrm{VO}_{2}$ during $\mathrm{EE}$ was not affected by previous RE. The differences among these studies may be related to the using of different devices in the aerobics (step, treadmill and cycle ergometer). These devices can promote an activation of different muscle mass and groups which can provide differences in the $\mathrm{VO}_{2}$. Also, in the Drummond et al. (2005), RE eliciting a similar muscle group than in the following $\mathrm{EE}$ may have influenced $\mathrm{VO}_{2}$ in the EE. Contrarily, in the Kang et al. (2009), the last RE performed before the EE was the shoulder press, eliciting a muscle group that not was involved in cycling. The higher values of $\mathrm{VO}_{2}$ observed in the EE reported by Kang et al. (2009) may be related to the physical level of the participants and not to the RE performed immediately before the EE. In the Drummond et al. (2005) investigation, the participants were physically active males involved in endurance 
Table 2 Mean $( \pm \mathrm{SD})$ of relative and absolute $\mathrm{VO}_{2}, \mathrm{HR}$ and RER during the strength training exercises in the two types of session.

\begin{tabular}{|c|c|c|c|c|c|c|}
\hline \multirow[b]{2}{*}{ Variables } & \multicolumn{3}{|c|}{$\begin{array}{c}\text { SCRE } \\
\text { Mean } \pm \text { SD }\end{array}$} & \multicolumn{3}{|c|}{$\begin{array}{c}\text { SRSRS } \\
\text { Mean } \pm \text { SD }\end{array}$} \\
\hline & Total & Block1 & Block2 & Total & Block1 & Block2 \\
\hline $\mathrm{VO}_{2}\left(1 \mathrm{~min}^{-1}\right)$ & $1 \cdot 02 \pm 0.21$ & $0.94 \pm 0.18$ & $1.09 \pm 0.24^{*}$ & $1 \cdot 12 \pm 0 \cdot 19$ & $1 \cdot 01 \pm 0.19$ & $1 \cdot 22 \pm 0 \cdot 20 *$ \\
\hline $\mathrm{VO}_{2}\left(\mathrm{ml} \mathrm{kg}^{-1} \min ^{-1}\right)$ & $17 \cdot 08 \pm 3 \cdot 25$ & $15 \cdot 60 \pm 2 \cdot 86$ & $18 \cdot 20 \pm 3 \cdot 44^{*}$ & $18 \cdot 21 \pm 2 \cdot 97$ & $16 \cdot 80 \pm 2.93$ & $19.95 \pm 2.94^{*}$ \\
\hline Heart Rate (bpm) & $152 \cdot 35 . \pm 13 \cdot 62$ & $143 \cdot 08 \pm 15 \cdot 64$ & $156.06 \pm 15.59^{*}$ & $146 \cdot 85 \pm 12 \cdot 39$ & $133 \cdot 67 \pm 10 \cdot 31$ & $159 \cdot 14 \pm 14 \cdot 70^{*}$ \\
\hline RER & $1.06 \pm 0.03$ & $1 \cdot 12 \pm 0.04$ & $1.05 \pm 0.02$ & $1.09 \pm 0.03$ & $1.09 \pm 0.04$ & $1.07 \pm 0.02$ \\
\hline
\end{tabular}

SCRE, session where the step choreography was performed continuously for 30 min before the resistance exercises blocks; SRSRS, session where the step choreography was performed in three blocks of 10 min before, in the middle and after the resistance exercises blocks; $\mathrm{VO}_{2}$, oxygen consumption; RER, rate exchange ratio; bpm, beats per min.

$* P<0 \cdot 0001$ between block 1 and block 2 .

Table 3 Mean $( \pm \mathrm{SD})$ of RMR and recovery $\mathrm{VO}_{2}$

\begin{tabular}{|c|c|c|}
\hline Variable & $\begin{array}{c}\text { SCRE } \\
\text { Mean } \pm \text { SD }\end{array}$ & $\begin{array}{c}\text { SRSRS } \\
\text { Mean } \pm \text { SD }\end{array}$ \\
\hline $\operatorname{RMR}\left(\mathrm{ml} \mathrm{kg}^{-1} \min ^{-1}\right)$ & $4 \cdot 40 \pm 0 \cdot 40$ & $4 \cdot 36 \pm 0.54$ \\
\hline Recovery $\mathrm{VO}_{2} 0-5 \min \left(\mathrm{ml} \mathrm{kg}^{-1} \min ^{-1}\right) *, * *$ & $12 \cdot 52 \pm 1 \cdot 64$ & $14 \cdot 63 \pm 1 \cdot 72$ \\
\hline Recovery $\mathrm{VO}_{2} 5-10 \mathrm{~min}\left(\mathrm{ml} \mathrm{kg}^{-1} \min ^{-1}\right) * *$ & $6 \cdot 41 \pm 1 \cdot 04$ & $6.95 \pm 0.83$ \\
\hline Recovery VO2 $10-15 \min \left(\mathrm{ml} \mathrm{kg}^{-1} \mathrm{~min}^{-1}\right) * *$ & $6.06 \pm 1.20$ & $6 \cdot 30 \pm 0 \cdot 81$ \\
\hline Recovery $\mathrm{VO}_{2} 15-20 \mathrm{~min}\left(\mathrm{ml} \mathrm{kg}^{-1} \mathrm{~min}^{-1}\right) * *$ & $5 \cdot 86 \pm 0.89$ & $6.23 \pm 0.79$ \\
\hline Recovery VO2 $20-25 \min \left(\mathrm{ml} \mathrm{kg}^{-1} \mathrm{~min}^{-1}\right) * *$ & $5 \cdot 68 \pm 0 \cdot 85$ & $6.02 \pm 0.78$ \\
\hline Recovery $\mathrm{VO}_{2} 25-30 \mathrm{~min}\left(\mathrm{ml} \mathrm{kg}^{-1} \mathrm{~min}^{-1}\right) * *$ & $5 \cdot 60 \pm 0 \cdot 84$ & $5.86 \pm 0.93$ \\
\hline
\end{tabular}

SCRE, session where the step choreography was performed continuously for 30 min before the resistance exercises blocks; SRSRS, session where the step choreography was performed in three blocks of 10 min before, in the middle and after the resistance exercises blocks; $\mathrm{VO}_{2}$, oxygen consumption; RMR, resting metabolic rate.

$* P=0.004$ between sessions.

$* * P<0.05$ between all recovery moments and RMR.

and strength training at least 2 days a week for 6 months and the subjects in Kang et al. (2009) were sedentary healthy males and females. Thus, participants with low physical fitness may influence the $\mathrm{VO}_{2}$ during the $\mathrm{EE}$ regardless of the type of $\mathrm{RE}$ used immediately before. To reinforce this idea, the lack of effect of RE before $\mathrm{EE}$ in Alves et al. (2012) and in the present study shows that the participants were trained in strength and aerobic training for at least 6 months and the RE used immediately before the EE did not involve muscle groups that are agonist during EE.

Another interesting aspect observed in the present study was that in the period of recovery (postsession), 0-5 min, $\mathrm{VO}_{2}$ was significantly higher in the SRSRS session when compared with SCRE. In Alves et al. (2012) and Drummond et al. (2005) studies, when the EE was performed after RE, it presented lower values of $\mathrm{VO}_{2}$ rec compared with other exercise sequences. This fact may be related to the time of $\mathrm{EE}$ in Alves et al. (2012) and in Drummond et al. (2005), which were 20 and $30 \mathrm{~min}$, respectively. Contrarily, in the present study, the time of EE was $10 \mathrm{~min}$. However, these minimal differences in the $\mathrm{VO}_{2}$ rec were likely not enough to promote the weight loss.

Moreover, the postexercise $\mathrm{VO}_{2}$ values observed by $\mathrm{Di}$ Blasio et al. (2012) were significantly higher when the EE was divided into three blocks of 10 min compared with when the same EE was performed continuously for $30 \mathrm{~min}$. We cannot compare the postexercise $\mathrm{VO}_{2}$ in the present study and in the Di Blasio et al. (2012) study because we used intervals of 5 min to analyse the dynamics of $\mathrm{VO}_{2}$ and Di Blasio et al.(2012) presented the overall values for an amounted period of $30 \mathrm{~min}$. The postexercise $\mathrm{VO}_{2}$ produced by the differences may be related to several physiological processes such as the elevation the cardiac activity; the lactate removal; the regulation of the body temperature; the replenishment of adenosine triphosphate and phosphocreatine muscle stores; the replacement of $\mathrm{O}_{2}$ in blood circulation and muscle; the glycogen resynthesize and the increase in the rate of the triglyceride - fatty acid cycle (Borsheim \& Bahr, 2003).

In summary, the results of the present study suggest that the sequence of the SC and RE, (before or divided between 
RE blocks), did not affect exercise and postexercise $\mathrm{VO}_{2}$, with an exception of the first $5 \mathrm{~min}$ of recovery. To use step choreography and resistance exercise in the same exercise session is a good strategy to promote an elevation of the $\mathrm{VO}_{2}$ during and after exercise. In addition, the SC can be performed continuously or divided throughout the overall time in blocks between the RE to promote alterations in the physiological demands. Thus, fitness trainers can use this strategy to streamline their classes without influencing the total session energy expenditure.

\section{Acknowledgments}

The authors express their gratitude to the participants who volunteered to make this study possible and also would like to thank to the Esposende 2000, E.M.

\section{Conflict of interest}

The authors declare no conflict of interest.

\section{References}

ACSM. American College of Sports Medicine. Guidelines for Exercise Testing and Prescription (2013); vol 9, part 7, pp. 162-190. Lippincott Williams \& Wilkins, Philadelphia.

Alves J, Saavedra F, Simão R, et al. Does aerobic and strength exercise sequence in the same session affect the oxygen uptake during and postexercise? J Strength Cond Res (2012); 26: 1872-1878.

Borsheim E, Bahr R. Effect of exercise intensity, duration and mode on post-exercise oxygen consumption. Sports Med (2003); 33: 1037-1060

Cadore EL, Izquierdo $\mathrm{M}$, Alberton $\mathrm{CL}$, et al. Strength prior to endurance intra-session exercise sequence optimizes neuromuscular and cardiovascular gains in elderly men. Exp Gerontol (2012); 47: 164-169.

Chtara M, Chamari K, Chaouachi $M$, et al. Effects of intra-session concurrent endurance and strength training sequence on aerobic performance and capacity. Br J Sports Med (2005); 39: 555-560.

Cohen J. Statistical power analysis for the behavioral sciences. In: The $\mathrm{T}$ test for Means (2013); vol.2, part 2, pp. 52-74. Academic press, New York, NY.

Davitt PM, Pellegrino JK, Schanzer JR, et al. The effects of a combined resistance training and endurance exercise program in inactive college female subjects: does order matter? J Strength Cond Res (2014); 28: 1937-1945.
Di Blasio A, Gemello E, Di Iorio A, et al. Order effects of concurrent endurance and resistance training on post-exercise response of non-trained women. J Sports Sci Med (2012); 11: 393-399.

Dolezal BA, Potteiger JA. Concurrent resistance and endurance training influence basal metabolic rate in nondieting individuals. J Appl Physiol (1998); 85: 695-700.

Drummond MJ, Vehrs PR, Schaalje GB, et al. Aerobic and resistance exercise sequence affects excess postexercise oxygen consumption. J Strength Cond Res (2005); 19: 332-337.

Ghahramanloo E, Midgley AW, Bentley DJ. The effect of concurrent training on blood lipid profile and anthropometrical characteristics of previously untrained men. J Phys Act Health (2009); 6: 760-766.

Kang J, Rashti SL, Tranchina CP, et al. Effect of preceding resistance exercise on metabolism during subsequent aerobic session. Eur J Appl Physiol (2009); 107: 43-50.

Karvonen J, Vuorimaa T. Heart rate and exercise intensity during sports activities. Sports Med (1988); 5: 303-311.

Kraemer WJ, Fry A, Ratamess N, et al. Strength Testing: Development and Evaluation of Methodology. (1995); vol 2, pp. 115-138. Human Kinetics, Champaign.

LeMura LM, von Duvillard SP, Andreacci J, et al. Lipid and lipoprotein profiles, cardiovascular fitness, body composition, and diet during and after resistance, aerobic and combination training in young women. Eur J Appl Physiol (2000); 82: 451-458.

Leveritt M, Abernethy PJ, Barry B, et al. Concurrent strength and endurance training: the influence of dependent variable selection. J Strength Cond Res (2003); 17: 503508.

McCarthy JP, Agre JC, Graf B, Pozniak MA, Vailas AC. Compatibility of adaptive responses with combining strength and endurance training. Medicine and Science in Sports and Exercise (1995); 27: 429-436.

Oliveira NL, Oliveira J. Excess postexercise oxygen consumption is unaffected by the resistance and aerobic exercise order in an exercise session. J Strength Cond Res (2011); 25: 2843-2850

Rosa C, Vilaça-Alves J, Fernandes HM, et al. Order effects of combined strength and endurance training on testosterone, cortisol, growth hormone and IGFBP-3 in concurrent-trained men. J Strength Cond Res (2015); 29: 74-79.

Schumann M, Eklund D, Taipale RS, et al. Acute neuromuscular and endocrine responses and recovery to single-session combined endurance and strength loadings:"order effect" in untrained young men. J Strength Cond Res (2013); 27: 421433. 